\title{
Development of a Three-Dimensional In Vitro Model for Longitudinal Observation of Cell Behavior: Monitoring by Magnetic Resonance Imaging and Optical Imaging
}

\author{
Klaus Kruttwig, ${ }^{1}$ Chantal Brueggemann, ${ }^{1}$ Eric Kaijzel, ${ }^{2}$ Susanne Vorhagen, ${ }^{1}$ \\ Thomas Hilger, ${ }^{3}$ Clemens Löwik, ${ }^{2}$ Mathias Hoehn ${ }^{1}$ \\ ${ }^{1}$ In-vivo-NMR-Laboratory, Max-Planck-Institute for Neurological Research, Gleuelerstrasse 50, 50931 Cologne, Germany \\ ${ }^{2}$ Department of Endocrinology, Leiden University Medical Centrum, Leiden, The Netherlands \\ ${ }^{3}$ Department of Radiation Therapy, University Clinics Cologne, Cologne, Germany
}

\begin{abstract}
Purpose: The aim of this study is the development of a three-dimensional multicellular spheroid cell culture model for the longitudinal comparative and large-scale screening of cancer cell proliferation with noninvasive molecular imaging techniques under controlled and quantifiable conditions.

Procedures: The human glioblastoma cell line Gli36 $\mathrm{EGFR}$ was genetically modified to constitutively express the fluorescence protein mCherry, and additionally labeled with iron oxide nanoparticles for high-field MRI detection. The proliferation of aggregates was longitudinally monitored with fluorescence imaging and correlated with aggregate size by light microscopy, while MRI measurements served localization in 3D space. Irradiation with $\mathrm{y}$-rays was used to detect proliferational response.

Results: Cell proliferation in the stationary three-dimensonal model can be observed over days with high accuracy. A linear relationship of fluorescence intensity with cell aggregate size was found, allowing absolute quantitation of cells in a wide range of cell amounts. Glioblastoma cells showed pronounced suppression of proliferation for several days following high-dose $\mathrm{Y}$-irradiation.

Conclusions: Through the combination of two-dimensional optical imaging and $3 \mathrm{D}$ MRI, the position of individual cell aggregates and their corresponding light emission can be detected. This allows an exact quantification of cell proliferation, with a focus on very small cell amounts (below 100 cells) using high resolution noninvasive techniques as a well-controlled basis for further cell transplantation studies.
\end{abstract}

Key words: Fluorescence imaging, Magnetic resonance imaging, Generation of mCherry expressing cells, Human glioblastoma cell line, Quantification of cells by fluorescence intensity, Proliferation dynamics of cell aggregates, Multimodality imaging, X-ray treatment of cancer cells

\section{Introduction}

$\mathrm{W}$ hen transgenic cell lines are generated with expression of imaging reporters and additional labeling for

Correspondence to: Mathias Hoehn; e-mail: mathias@nf.mpg.de magnetic resonance imaging (MRI), their proliferation characteristics need to be investigated under well-defined control conditions before in vivo transplantation experiments which enable detailed understanding of in vivo processes. In the past, such investigations have been performed on monolayer cultures. However, a three-dimensional cell 
aggregate system is preferable allowing a more realistic characterization of cell dynamics and complex pathophysiological responses to (therapeutic) interference with proliferation. Formation of multicellular aggregates has been reported for different cell types and tissues in vitro without loss of tissue-like characteristics [1]. This culture form resembles functional tissue properties better than two-dimensional culture variants. Recent advances in 3D spheroid research include new functional methods like biochip-based culture systems for high-throughput drug screening [2]. In the field of imaging research, the identification, observation, and exact quantification of variable and very small cell amounts (below 100 cells) is an emerging topic.

Molecular imaging is a very sensitive and versatile tool for the detection of different kinds of cells and of cellular characteristics with wide application in tumor biology. Whole body imaging techniques, either fluorescence, bioluminescence, or magnetic resonance, provide powerful tools for noninvasive, longitudinal monitoring of tumor cell progression in small animal in vivo models [3]. The combination of optical imaging techniques with high-field MRI generates a synergy provided by the high sensitivity of optical imaging and the high, three-dimensional resolution of MRI [4], resulting in the detectability of cells within an object. Cells can be labeled intracellularly by endocytosis of superparamagnetic particles containing iron oxide, coated by a polymer or polysaccharide shell [5]. The iron oxide nanoparticles effectively shorten the transverse relaxation time $T_{2}$ of protons through susceptibility-induced local magnetic field inhomogeneities, resulting in hypointensity contrast in $\mathrm{T}_{2}$-weighted $\mathrm{MR}$ images [6]. The overall diameter of particles used for cell labeling studies ranges from $30 \mathrm{~nm}$ up to or even larger than $1 \mu \mathrm{m}$ [8].

High-grade malignant glioblastoma is one of the most common primary tumors of the central nervous system and is characterized by highly extensive tissue infiltration and high proliferation rates. Due to its aggressiveness the mean survival time of patients is less than 12 months [1-3]. The glioblastoma cell line Gli36 $\triangle \mathrm{EGFR}$, formerly established by retroviral transfer of a mutant epidermal growth factor receptor (EGFR) into the Gli36 glioblastoma cell line [7], was chosen as the model cell line for our in vitro investigation on cell proliferation under controlled conditions. $\triangle$ EGFR-transduced tumor cells are excellent models for in vivo applications because of their rapid growth in rat brains [10]. We introduced the fluorescent protein mCherry under control of the cytomegalovirus promoter, resulting in a strong expression of this optical imaging reporter. Additionally, the cells were labeled with the clinically used MRI iron oxide contrast agent Endorem, producing a pronounced $\mathrm{T}_{2}{ }^{*}$-weighted contrast.

In the present study, we report establishment of a cell aggregate model which is suitable for a detailed and longitudinal characterization of glioma cell proliferation and for large-scale screening purposes under controlled conditions in vitro. For this purpose, we have developed a three-dimensional, agarosebased in vitro cell model, while applying combined imaging techniques for complementary information content. It was the aim of the study to use optical imaging (fluorescence in the present case) and phase contrast microscopy for the sensitive detectability of small cell clusters and achieve the quantification of even small cell aggregates. High-field MRI was chosen for precise three-dimensional localization of the selected cell aggregates. We have deliberately chosen to set up a cell aggregate model for our investigation of tumor cell behavior as these aggregates represent a more complex cellular model, compared to a monolayer-culture model. To demonstrate the exemplary potential of this cell model, the evaluation of cancer treatment strategies was investigated monitoring the response of tumor cell aggregates to $\gamma$-ray exposure.

\section{Materials and Methods}

\section{Cell Culture}

Gli36 $\triangle$ EGFR human glioblastoma cells $[7,8]$ were kindly provided by A. Jacobs (MPI for Neurological Research, Cologne). Cells were maintained in Dulbecco's modified eagles medium, supplemented with $10 \%$ fetal calf serum (FCS), penicillin $\left(100 \mathrm{U} \mathrm{ml}^{-1}\right)$, streptomycin $\left(100 \mu \mathrm{g} \mathrm{ml}^{-1}\right)$, and $200 \mu \mathrm{M}$ L-glutamine (all reagents from PAA, Pasching, Austria) on cell culture dishes (Greiner, Frickenhausen, Germany). All cells were maintained in $5 \% \mathrm{CO}_{2}$ and $90 \%$ humidity at $37^{\circ} \mathrm{C}$.

\section{Generation of mCherry Expressing Gli364EGFR Clones}

The fluorescence reporter mCherry was kindly provided by $\mathrm{R}$. Tsien (University of California, San Diego, CA, USA). The mCherry containing plasmid was treated with BamHI and EcoRI (New England Biolabs, Ipswich, USA) and introduced into the expression vector pcDNA3.1(+) downstream of the cytomegalovirus promoter (Invitrogen, Karlsruhe, Germany). Glioblastoma cells were transfected using the lipofection agent Metafectene (Biontex Laboratories, Martinsried, Germany) and $1 \mu \mathrm{g}$ of the purified plasmid for $4 \times 10^{5}$ cells. Further selection was performed with the supplementation of $400 \mu \mathrm{g} \mathrm{ml} \mathrm{g}^{-1}$ neomycin (PAA). For the generation of a homogeneous population, single cells were cultured in 96-well plates (Greiner) and screened for fluorescence intensities. Clone number 17 was chosen for the following experiments because of its high mCherry fluorescence.

\section{Aggregate Culture and Cellular Labeling}

For the formation of the cellular aggregates, cells were cultured on cell culture dishes coated with Poly(2-hydroxyethyl methacrylate) (Poly-HEMA, Sigma-Aldrich, Taufkirchen, Germany) preventing attachment to the surface. The cells were labeled by co-incubation with the clinically used MRI contrast agent Endorem ${ }^{\circledR}$ (Guerbet, Roissy, France) consisting of small superparamagnetic iron oxide particles with a mean diameter of $150 \mathrm{~nm}$ and a total iron content of $11.2 \mathrm{mg} \mathrm{ml}^{-1}$ [9] during the spontaneous aggregate formation. Incubation with $112 \mu \mathrm{g} \mathrm{ml}{ }^{-1}$ or $224 \mu \mathrm{g} \mathrm{ml}{ }^{-1}$ Endorem was performed for $24 \mathrm{~h}$ in a total volume of $1 \mathrm{ml}$ FCS-containing medium and $4 \times 10^{5}$ cells. At the end of the labeling experiment, 
cells were washed three times with phosphate-buffered saline (PBS) without $\mathrm{Ca}^{2+}$ and $\mathrm{Mg}^{2+}$ (PAA), and centrifuged at $16 \times g$ in $15-\mathrm{ml}$ tubes. Aggregates were suspended in FCS-containing medium without phenol red (Invitrogen) and either plated for $3 \mathrm{~h}$ on PolyL-ornithine and $1 \mu \mathrm{g} \mathrm{ml}^{-1}$ fibronectin (Sigma-Aldrich) coated cover slips for Prussian blue staining and immunocytochemistry or for incubation experiments in low-melting agarose (Sigma-Aldrich) phantoms. We used $35 \mathrm{~mm}$ diameter dishes equipped with a $2 \mathrm{~mm}$ bottom grid for colocalization purposes (Thermo Fisher Scientific, Langenselbold, Germany). For the imaging experiments, aggregates were seeded in low-melting agarose and overlayed with FCScontaining cell culture medium. In some staining experiments aggregates were placed on treated four-well chamber slides (Becton Dickinson, Heidelberg, Germany).

For the determination of a potential influence of the MRI contrast agent on the fluorescence measurement, $1 \times 10^{5}$ cells were cultured in 24-well plates (Greiner) in the presence of 112 or $224 \mu \mathrm{g} \mathrm{ml}{ }^{-1}$ Endorem. Fluorescence analysis was performed $24 \mathrm{~h}$ after the initial supplementation and further washing steps in a multimode reader (Mithras LB 940, Berthold Technology, Bad Wildbad, Germany).

\section{Immunocytochemistry and Light Microscopy}

Fixation of aggregates cultured on cover slips was performed for $25 \mathrm{~min}$ with a $4 \%$ paraformaldehyde solution at room temperature, and agarose embedded aggregates were fixated for $24 \mathrm{~h}$ at $4{ }^{\circ} \mathrm{C}$. After permeabilization with $0.25 \%$ Triton X-100 (Fluka, Taufenkirchen, Germany) and further incubation with a $5 \%$ bovine serum albumin (Fraction V Cohen, PAA) cells were incubated with the primary antibodies anti-vimentin (mouse; 1:1,000, Sigma Aldrich), and FITC coupled anti-dextran (1:25, clone DX1, Stem Cell Technologies, Vancouver, Canada). For anti-vimentin antibody detection, the secondary antibody anti-mouse 488 (Thermo Fisher Scientific) was used. Nuclei were detected with the bisbenzimide Hoechst Dye 33342 (1:1,000, Sigma Aldrich) or with Sytox green (1:1,000, Invitrogen) after 10 min incubation. The glass cover slips were embedded with Aquamount (Polyscience Inc., Eppelheim, Germany) and further processed.

Fluorescence and confocal microscopy were performed on a Leica Laser scanning microscope (LSM) equipped with a supplementary CCD-camera (Leica, Wetzlar, Germany). Phase contrast microscopy was recorded on a Zeiss Axiovert microscope (Zeiss, Goettingen, Germany). For the determination of the aggregate diameter, the minimal and maximal in-plane diameters of each multicellular aggregate were measured with phase contrast microscopy and coregistered with the fluorescence images. The mean diameter of the two was used for the volume calculation.

For the detection of iron, cells were treated with a diaminobenzidine (DAB) enhanced Prussian blue staining. Cells were fixated, washed in PBS, and incubated for $30 \mathrm{~min}$ in a 1:1 ratio solution with $2 \%$ potassium ferrocyanide (Perl's reagent, Merck, Darmstadt, Germany) and $2 \% \mathrm{HCl}$, followed by $10 \mathrm{~min}$ DAB treatment (SigmaAldrich) according to the manufacturer's protocol.

\section{Fluorescence Imaging}

Fluorescence imaging was performed with the real-time fluorescence and bioluminescence small animal optical imaging system, Photon Imager (Biospace Labs, Paris, France), equipped with a custom-manufactured platform including four glassfibers for optimal illumination of the cell culture sample. Fluorescence light exposure time was $30 \mathrm{~s}$, the filters were set for emission at $610 \mathrm{~nm}$ and excitation at $585 \mathrm{~nm}$. Aperture was set to 2.8. The optical imaging experiments of the $35 \mathrm{~mm}$ cell culture dishes were performed with open lids to reduce reflections.

\section{Magnetic Resonance Imaging}

All magnetic resonance imaging experiments were performed on a Bruker Biospec 11.7 T dedicated animal scanner (Bruker BioSpin, Ettlingen, Germany) with a $16 \mathrm{~cm}$ horizontal bore magnet, equipped with actively shielded gradients $\left(750 \mathrm{mT} \mathrm{m}^{-1}\right)$. A transmit-receive radiofrequency coil with a $45 \mathrm{~mm}$ diameter was used. Threedimensional high-resolution $\mathrm{T}_{2}{ }^{*}$-weighted $\mathrm{MR}$ images were acquired using a gradient echo sequence (FLASH, TR=100 ms, $\mathrm{TE}=5 \mathrm{~ms}$, or $\mathrm{TE}=18 \mathrm{~ms}$, flip angle $30^{\circ} ; 12$ averages). The field-ofview was $36 \times 37 \times 5 \mathrm{~mm}^{3}$ with a matrix size of $512 \times 512 \times 64$, resulting in an almost isotropic spatial resolution of $70 \times 74 \times 78 \mu \mathrm{m}$.

\section{Radiation Exposure Treatment}

Cell systems were irradiated by high-energy photon radiation, generated by an electron linear accelerator (Type SL 75/5, Elekta, Crawley, GB). High energetic electrons are decelerated by a tungsten target, thereby producing bremsstrahlung of $5 \mathrm{MeV}$ energy at maximum. The radiation field was $20 \times 20 \mathrm{~cm}$, ensuring a homogeneous dose distribution across the whole sample holder. The distance between sample and radiation source was $100 \mathrm{~cm}$. Additionally, $2 \mathrm{~cm}$ of polymethyl methacrylate were positioned directly above the samples in order to eliminate the build-up effect. The agarose-based cell systems were irradiated with 20 and 50 Gy, respectively. Control cell systems without treatment were placed outside the treatment room during activation of the beam. After radiation exposure photon flux was determined over a time period of 6 days. In these experiments the fluorescence excitation intensity was reduced to $50 \%$.

\section{Data Processing}

Optical imaging experiments were analyzed with the software package PhotovisionPlus (Biospace Labs, Paris, France) and NIH imageJ. MRI images were processed using Paravision 4 (Bruker BioSpin, Ettlingen, Germany). 3D reconstruction was performed with the software package Amira (version 3.1, Mercury Computer Systems, Inc., Chelmsford, USA).

Statistical analysis was performed with Origin software package, version 8 (OriginLab Corporation, Northampton, USA).

\section{Results}

\section{Effect of Endorem Labeling}

Culturing cells in the presence of two different concentrations of the MRI contrast agent Endorem on an antiadhesive surface leads to an aggregate formation with minimal diameters ranging between 10 and $60 \mu \mathrm{m}$, as well as single cells. For detection of iron oxide particles in cells and for assessment of the integrity of the cytoskeleton after uptake of superparamagnetic particles, aggregates were placed for $3 \mathrm{~h}$ on a Poly-L-ornithine and fibronectin-coated 
surface (Fig. 1). The anti-dextran antibody staining shows a remarkable uptake of contrast agent particles in the cytoplasm as well as iron containing beads attached to the outer membrane surface of cells (Fig. 1c). Also, a clustering of the nanometer-sized particles could be observed. Single cells that migrated away from the aggregate center during the culturing period had intact, vimentin-positive intermediary filaments, and the mCherry protein was located in the cytoplasm as well as in the cell nucleus. Complementary to fluorescence microscopy, achieved with anti-dextran antibody for nanoparticle detection, Prussian blue staining (Fig. 1d) was performed to reveal the presence of iron with light microscopy: iron containing particles were found to be distributed inside the cells, but with a variation of quantitative uptake of particles. This variability was observed also in monolayer cultures after exposure to the contrast agent (data not shown). We therefore do not believe that such an inhomogeneous distribution is due to the chosen aggregate culture technique.

Cells, cultured as a monolayer and labeled for $24 \mathrm{~h}$ with two different concentrations of Endorem, showed the same mCherry fluorescence light intensity, independent of the supplemented contrast agent concentration (Fig. 2). Thus, additional incorporation of the MRI contrast agent does not lead to any fluorescence quenching effect.

\section{Coregistration of Fluorescence and MR Images}

After placing multicellular aggregates in an agarose-based matrix, enriched with serum-containing medium, photon emission of these phantoms was registered with the optical imager after excitation at $585 \mathrm{~nm}$ for $30 \mathrm{~s}$ (Fig. 3a), followed by recording of $\mathrm{T}_{2}{ }^{*}$-weighted $3 \mathrm{D}$ MRI scans (Fig. 3b). All of the stationary aggregates were detected and co-localized with these two independent imaging techniques. Due to the 3D capability of MRI, the position of the cell aggregates could also be discriminated along the third dimension (Fig. 3c).

For multimodal imaging coregistration, regions of interest were selected and analyzed (Fig. 4). Fluorescence imaging and phase contrast microscopy, performed for the analysis of minimal aggregate diameter, were determined (Fig. 4a, b). In a comparative analysis between two different $\mathrm{T}_{2} *$-weighted echo times (TE=5.0 and $18.0 \mathrm{~ms}$; Fig. 4c, d), each aggregate was clearly visible and could be coregistered with the microscopic pictures and the fluorescence images. Additionally, a 3D model of the aggregates (Fig. 4e), based on the MRI data of the $5 \mathrm{~ms}$ TE scan (Fig. 4c), displays the localization of cells at variable depths in the agarose phantom.

\section{Quantification of Fluorescence Signal as a Function of Cell Number and Aggregate Diameter}

Photon flux and measured in-plane diameters of various aggregates were determined from 200 randomly selected multicellular aggregates in 14 independent phantoms. This led to an excellent linear correlation (regression coefficient $R=0.9$ ) between estimated aggregate volume and fluorescent
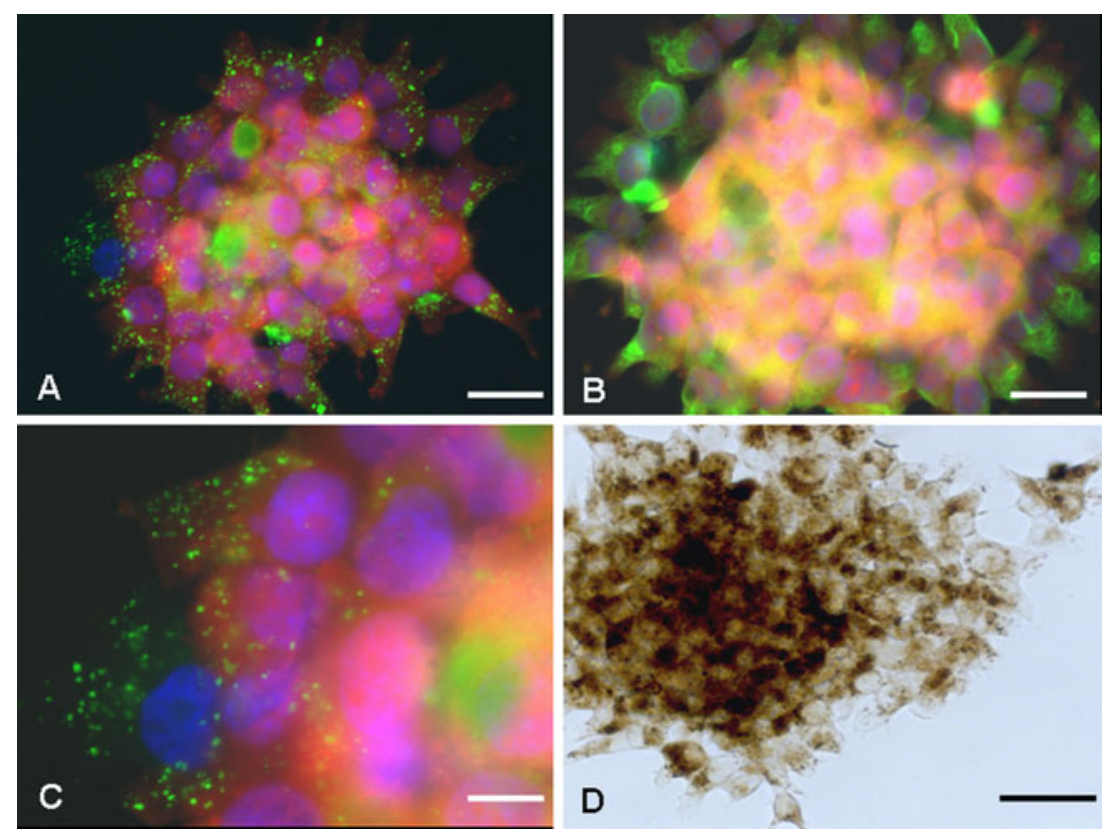

Fig. 1. Immunocytochemistry of multicellular aggregates in the agarose-based phantom. Aggregates were cultured on an adhesive surface and stained for the presence of dextran (green) (a, c) and vimentin (green) (b) to detect intermediary filaments. mCherry expression is displayed in red and the nuclei are stained with Hoechst Dye 33342 (b/ue). The distribution of the superparamagnetic beads inside cells, after $24 \mathrm{~h}$ exposure to the MRI contrast agent Endorem, displays on a DAB-enhanced Prussian blue staining (d) that individual cells can be effectively labeled in this culture model. Scale bars a, b, $25 \mu \mathrm{m}$; c $10 \mu \mathrm{m}$; d $50 \mu \mathrm{m}$. 


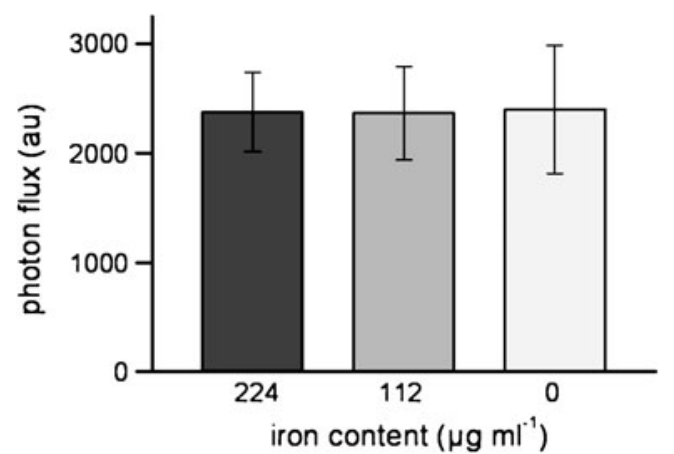

Fig. 2. Assay of the effect of incorporated iron particles on the fluorescence intensity. Cells were treated with different concentrations, equivalent to iron content, of the MRI contrast agent Endorem for $24 \mathrm{~h}$. Fluorescence detection analysis shows no discernable difference in photon detection between unlabeled and labeled cells. Number of independent experiments, $n=3$.

light intensity, as shown in Fig. 5. Fluorescent light intensity (FI) was found to depend on cluster volume with the following equation:

$$
F I=(39601 \pm 5064)+(6.32 \pm 0.13) \times V
$$

where $V$ is the volume of the cell aggregate under consideration. This volume was calculated from the measured cell aggregate diameter under the assumption of a perfect spherical cell distribution of the aggregate. In a small sampling study, we correlated the absolute cell number, determined with confocal laser scanning microscopy, with the photon flux, measured by the optical imager. The smallest detectable cell amount, clearly discriminated from the fluorescent background signal, was five cells. The relation between the fluorescent signal intensity and the cell number also follows a linear function (data not shown).

\section{Dynamics of Cell Aggregate Growth and Proliferation}

Cells were cultured for a time period of 5 days in an agarosebased, growth factor enriched matrix. Each day, photon flux and diameter of the aggregates (by light microscopy; see above) were determined (Fig. 6). A representative analysis of the photon flux of four aggregates with different diameters at the beginning of observation displays the proliferation of the selected stationary cell clusters (Fig. 6c). The diameter of the smallest of the four selected aggregates increased from $49.5 \mu \mathrm{m}$ at the beginning to $121.75 \mu \mathrm{m}$ after $120 \mathrm{~h}$. The aggregate with an initially larger diameter of $76.75 \mu \mathrm{m}$ showed a diameter of $176.75 \mu \mathrm{m}$ at the termination of the experiment.

\section{Monitoring Cell Aggregate Response to Radiation Treatment}

To investigate cancer therapy with $\gamma$-irradiation in vitro under controlled conditions, cellular aggregates were exposed to doses of 20 and $50 \mathrm{~Gy}$, respectively, at $24 \mathrm{~h}$ after setting up the cell culture. For the determination of radiation-induced effects the phantoms were cultured for the following 6 days. During this observation period, fluorescent light intensity was determined from a series of cell aggregates. At $24 \mathrm{~h}$ after irradiation, a slight decrease in photon flux was noted (Fig. 7). Proliferation of treated aggregates came to an almost complete halt, with a minute increase at the time point $90 \mathrm{~h}$ after seeding. After this time, a small decrease in photon flux was observed, indicating a slow but continuous loss of cells. Determination of aggregate diameters at the termination of the experiment led to a proliferative volume increase to $238 \% \pm 48 \%$ in the nonirradiated control group, when compared to the initial
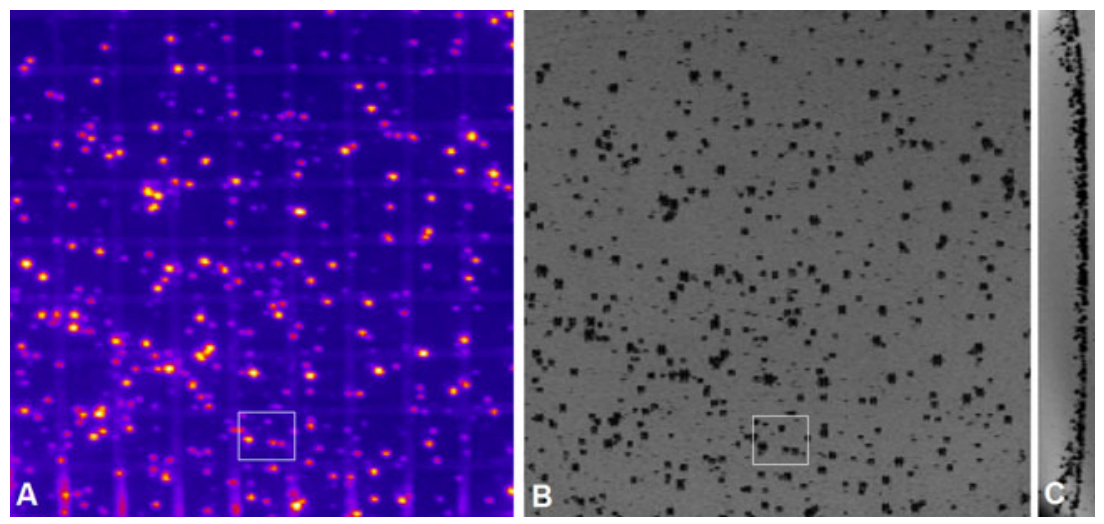

Fig. 3. Fluorescence image of multicellular aggregates with different amounts of cells in a three-dimensional agarose matrix (a). An exposure time of $30 \mathrm{~s}$ was sufficient to visualize the cellular clusters. For the 3D localization of the cells, pre-incubated with the MRI contrast agent Endorem, a $\mathrm{T}_{2}{ }^{*}$-weighted MRI scan was performed (b, c) on the same sample, following the fluorescence measurement. The coregistration of all cell clusters with both imaging modalities is notable. The indicated box uses the selected region-of-interest for further detailed analysis, as demonstrated in Fig. 4. The MRI projection perpendicular to the agarose phantom surface shows the depth distribution of the cell clusters (c). The grid dimension, visible in the fluorescence image, is $2 \mathrm{~mm}$. 

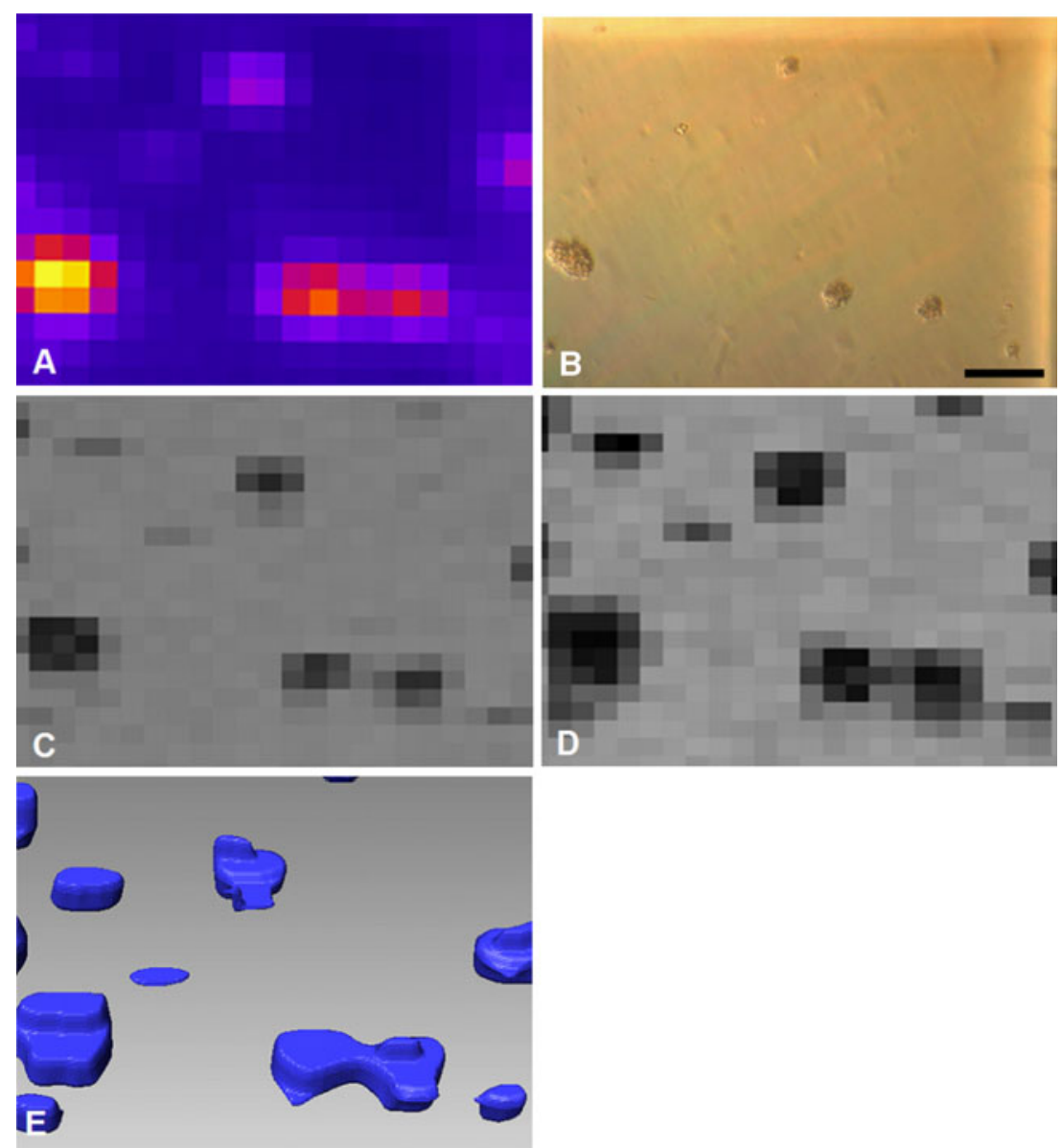

Fig. 4. The region-of-interest, demarcated in Fig. 3, shows the zoomed, highly resolved arrangement of a few cell clusters with the three independent imaging techniques: fluorescent imaging (a), phase contrast microscopy (b), and $\mathrm{T}_{2}{ }^{*}$-weighted 3D-MRI, with echo time TE of $5 \mathrm{~ms}$ (c) and $18 \mathrm{~ms}$ (d). A 3D model, based on the MRI data of the TE=5 ms scan, allows to discriminate the localization of cells along the axis perpendicular to the agarose surface plane (e). Scale bar $100 \mu \mathrm{m}$.

diameter at the experimental start. During the same observation period, the photon flux of the 20 Gy irradiated phantoms increased to $128 \% \pm 10 \%$, and for the cells treated with 50 Gy to $122.6 \% \pm 48.8 \%$ of the initial volume. The growth characteristics of the untreated control aggregates corresponded to the values obtained in proliferation experiments, visualized in Fig. 6.

\section{Discussion}

We have developed a stationary cell model system for the in vitro monitoring and quantification of cell dynamics. This model allows observation of cell proliferation under controlled conditions while assuring the cellular viability. Consequently, this opens the way for longitudinal studies with multiple measurements using even complementary imaging techniques. As these imaging techniques are also applicable for in vivo investigations, in vitro results have a clear translational potential. To our knowledge, this is the first description of a cell model system in which continuous monitoring of cellular characteristics over time and func-

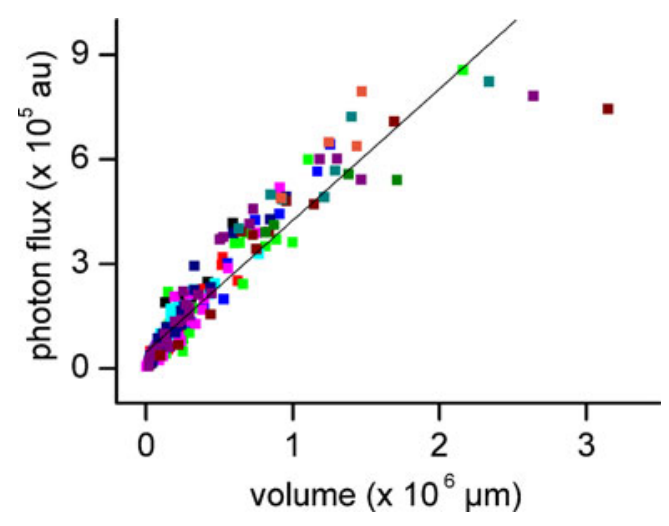

Fig. 5. Correlation between spherical aggregate volume, estimated from cluster diameter data of light microscopy, and fluorescence photon flux (recorded with the optical imager) of the aggregates. Different colors and symbols represent independent measurements or arbitrarily chosen clusters within one phantom. In some cases, separate, independent measurements of the same phantom were performed, and also of various different phantoms. The line reflects the fit of a linear function to all the data. 

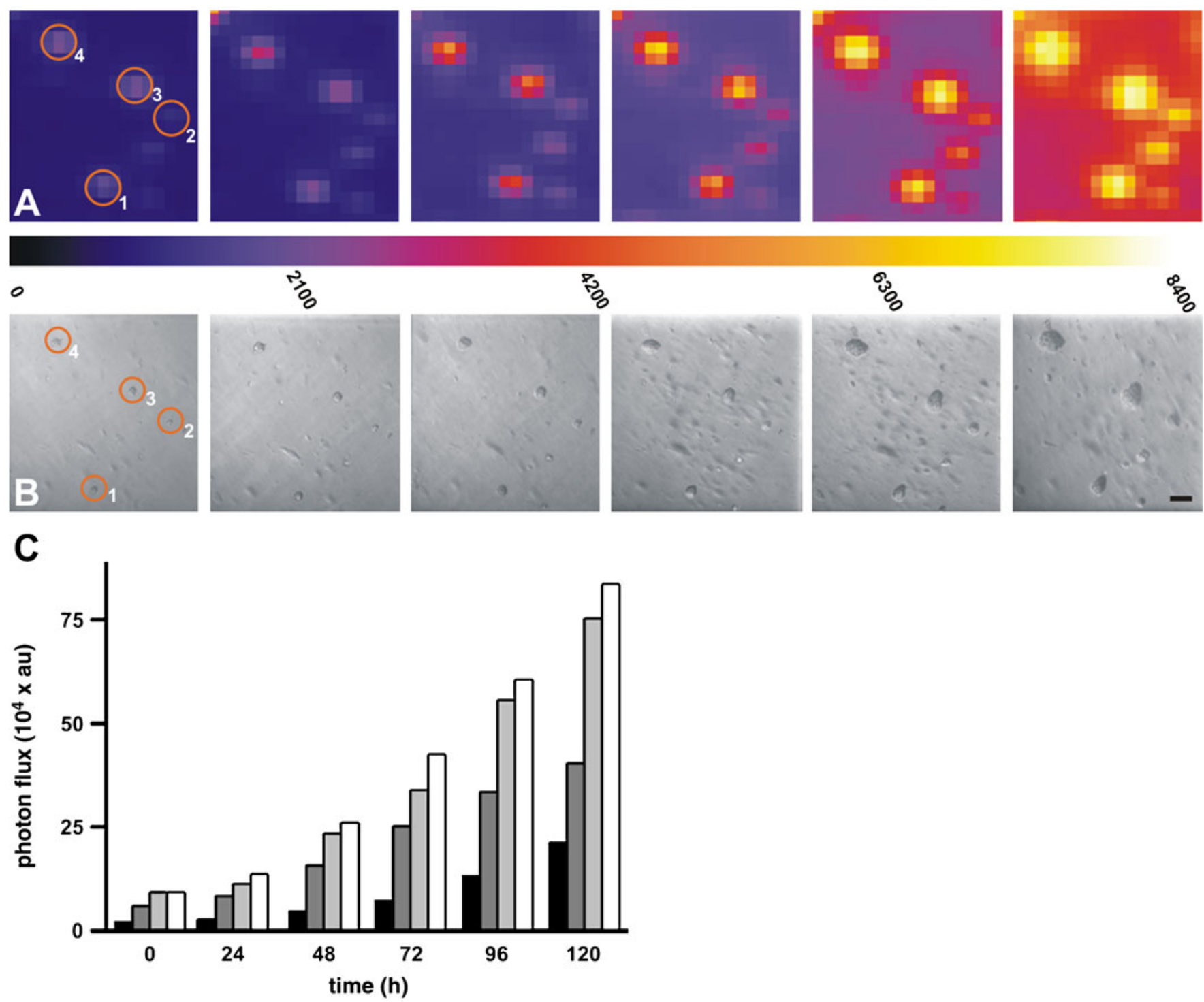

Fig. 6. Longitudinal monitoring of four selected cell aggregates over a time period of 5 days (circles labeled 1 to 4 in a). The upper row shows the fluorescence photon flux of the cell aggregates, with increasing intensity during continuing proliferation (i.e. cell cluster growth). The color bar displays the fluorescence intensity values of the photon flux. The corresponding phase contrast microscopic images (second row, b) indicate the cell aggregate growth in correspondence with the photon flux. Scale bar represents $50 \mu \mathrm{m}$. Quantitative measurement of the photon flux for the four cell aggregates over the observed time period is displayed in the bar diagram (c).

tional allocation to specific cell amounts is achieved with a novel multimodal imaging approach.

\section{Detectability of Small Cell Clusters}

Bioluminescence is the most commonly used in vivo imaging modality for signals coming from cells deep in the tissue. Available fluorescence reporters, on the other hand, especially GFP and EGFP, have serious limitations concerning the tissue penetration depth and because of severe autofluorescence contributions [10]. The red fluorescence protein and its even more red-shifted variants such as the mCherry protein from the fruit series [11], as well as the development of near-infrared dyes have longer excitation and emission wavelengths, thereby decreasing the contribution of autofluorescence substantially. More important, tissue penetration depth of light is substantially increased to deeper layers using these red-shifted probes for their lower tissue absorption and light scattering [12]. In the present investigation, the Gli36 $\triangle$ EGFR cells, displaying a highly invasive phenotype and characterized by rapid proliferation [7, 8], were transfected to express the fluorescence imaging reporter mCherry. We selected a clone with a mCherry expression 3.5 times stronger than the average value of the original neomycin-resistant mCherry-positive population. Exclusively, this cell line with the strong and stable mCherry expression was used for further experiments to assure maximal fluorescence detection sensitivity. 


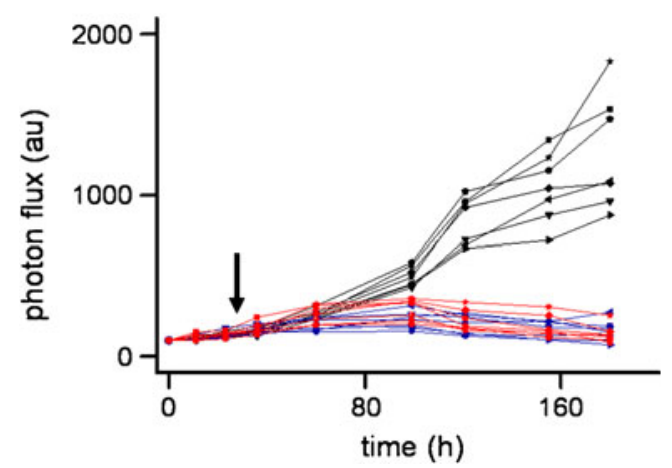

Fig. 7. Radiation treatment of embedded, cultured glioblastoma cells. Treatment with 20 Gy (red symbols) and 50 Gy (blue symbols) was performed at $24 \mathrm{~h}$ after seeding the cell aggregates in the matrix (arrow). The irradiated cells show a decreased photon flux while the untreated control group of cells (black symbols) demonstrates the typical growth behavior, in correspondence to the pattern shown in Fig. 6c.

For the MRI experiments, incubation with a concentration of 112 or $224 \mu \mathrm{g} \mathrm{ml}^{-1}$ Endorem for a time period of $20 \mathrm{~h}$ was found sufficient for detection of labeled cells by MRI. Detectability of small cell clusters was found to be variable in each separate sample preparation due to a nonhomogeneous uptake of the superparamagnetic particles among cells. Detectability of proliferating, growing cell clusters, on the other hand, was not observed to be compromised by proliferation-dependent dilution of the MRI label. As the clusters grow due to their proliferation, larger, compact cell aggregates arise, commonly easier visualized on MRI images. Detectability of labeled stem cells as a function of their proliferation was recently reported from our lab [9]. There it was found that a dilution factor of 256 (equivalent to a label dilution due to eight cell generations of proliferative duplication) still results in a clearly detectable MRI contrast in vitro.

\section{Quantification of Cell Cluster Size}

One difficulty with in vitro MRI is the determination of the number of labeled cells from the experimentally observed $T_{2}$ or $\mathrm{T}_{2} *$ signal decrease in a sample. Cell labeling with large particles (several hundred nanometers up to $1 \mu \mathrm{m}$ ) increases the MRI contrast enhancement through greater amounts of iron per particle but possesses a reduced cellular labeling efficiency compared to particles with a diameter around $100 \mathrm{~nm}$ [13]. One possibility to measure a small amount of cells or even single cells is to produce a highly diluted agarose-cell solution, in which the single hypointense spots on the MR images represent single cells (or small clusters thereof) serving direct cell quantification $[14,15]$. Culture of cells and multiple MRI measurements provides detailed information about cellular activity and behavior. Therefore, growth and invasion characteristics of multicellular melanoma tumor spheroids with larger diameters were analyzed in agarose gels [16]. A recent publication from Huang and colleagues describes the expansion dynamics of glioma cells embedded in gadolinium-DTPA enriched collagen I over the time period of $12 \mathrm{~h}$ with high spatial resolution [17]. These cells were not previously labeled with an MRI contrast agent, which would, however, be a necessary prerequisite for further in vivo transplantation experiments. Bernas et al. labeled C6 rat glioma cells with fluorescent, iron-containing particles that had a mean diameter of $0.9 \mu \mathrm{m}$ [18]. Labeled cells in this study were cultured for up to 8 days, allowing to invade into a collagen I matrix, and further analyzed with a $1.5 \mathrm{~T}$ MRI and corresponding phase contrast microscope. All the above cited studies have some limitations in common concerning their labeling techniques. Large amounts of nanometer-sized particles are necessary for efficient and robust detection of small cell amounts, but homogeneous, equal labeling of all cells is not guaranteed. A further problem is a dilution process of the iron oxide particles with each cell division, leading to contrast agent dilution, possibly even beyond detectability [8]. Therefore, quantification of the exact cell amount in a longitudinal study is problematic with these MRI label-based methods.

We chose a stationary proliferation model allowing multicellular aggregates to proliferate and to be analyzed with different multimodal imaging techniques with the focus on the detectability and colocalization. This allows a rapid screening and identification of multicellular clusters while observing individual cell cluster dynamics in longitudinal studies. Stable expression of the fluorescence marker mCherry allows determination of the exact cell number in a sample with high sensitivity, without problems due to contrast agent dilution effects and inhomogeneous uptake. We calculated, based on confocal LSM, the minimal detectable cell amount of various cell clusters and found a linear correlation to the photon flux (K. Kruttwig et al., unpublished results). For larger cell amounts and fast screening of the samples, it is useful to determine the microscopic cluster diameter instead of the absolute cell amount, as the latter approach requires time-consuming laser scanning microscopy. The optical microscopic methods are limited by axial resolution and working distances of the objectives $[18,20]$. Fluorescence imaging is a rapid method for the detection of small amounts of living cells with high sensitivity [19], but without in-plane high resolution and without information about the axial distribution. As seen in Fig. 6 for larger cell aggregate volumes, the experimental data deviates slightly from the linear behavior for larger cell cluster volumes. This is indication that the assumption of a perfectly spherical volume no longer holds for large cell clusters which will spread out to reach more and more a disk-shaped volume. Here, three-dimensional information about the axial cell distribution will come in handy: highresolution MRI provides the possibility to acquire 3D data sets with resolution of $100 \mu \mathrm{m}$ or better [20], but at the expense of longer measurement periods compared to photon imaging. The cells were detected and quantified with 
fluorescence imaging (at a scan time of $30 \mathrm{~s}$ ), and were visualized with a resolution of $70 \mu \mathrm{m}$ with MRI. Where necessary, further 3D image reconstruction based on the MRI data could be added for detailed localization issues. As shown in Figs. 3, 4, and 6, it is easily possible to discriminate the aggregates with both imaging techniques with high resolution and in longitudinal studies.

\section{Radiation Response Monitoring}

A radiation induced decrease of cell proliferation of the human glioblastoma cell line was observed after irradiation with lethal dosages of either 20 or $50 \mathrm{~Gy}$. This was visualized with quantitative fluorescence imaging. Glioblastoma cells are known to show a markedly augmented radioresistance to radiation therapy strategies and often respond with an enrichment of a $\mathrm{CD} 133^{+}$cancer stem cell population [21, 22]. After irradiation with sublethal doses an enhancement of migrational activity and infiltration has been reported [23].

In a recently published study from Thorsen et al. [24], a specially designed dish for radiosurgery of human glioma spheroids in suspension was used for irradiation with 12 or 24 Gy using a gamma knife. These authors reported a dosedependent inhibition of tumor growth after irradiation, observed with microscopic techniques. The radiation effects on the tumor growth of pretreated tumor cells was assessed by MRI after xenotransplantation into the nude rat brain.

The combination of both imaging techniques for the application to our in vitro cell model system provides an ideal tool for rapid noninvasive monitoring of the cell dynamics of large amounts of tumor cells (in various cell aggregates). This investigation procedure therefore makes the cell system useful for the assessment of cancer treatment efficiency by using proliferational activity as the evaluation criterion. In principle, cell clusters with different response behavior to a treatment modality (e.g. radiation) can be identified and analyzed separately to better understand involved pathomechanisms.

Additional transfection of the cells with luciferase to allow use of bioluminescence imaging can be an additional method to monitor and quantify metabolically active cells due to the ATP dependence of the enzymatic reaction [2527]. Compatibility of the agarose model with such vitality marker strategy has already been demonstrated in a proofof-principle experiment in our laboratory (K. Kruttwig, unpublished).

\section{Summary}

We have developed a stationary cell model system, allowing observation of viable cellular dynamics, compatible for longitudinal studies. The fluorescence imaging method has been calibrated demonstrating a linear correlation between fluorescence intensity and volume of the cell aggregates based on light microscopy and photon flux determination.
This now permits characterization of the cell aggregate dynamics in a quantitative manner in vitro with noninvasive imaging techniques. Direct application to radiation treatment of the human glioblastoma cell model, used in our present investigation, shows that a sensitive recording of radiationinduced proliferation modulation is possible with this cell model system. On a more general level, the here described setup can be applied to systematic characterization of cell line behavior under different conditions, and can be exploited to assess efficiency of treatment strategies under controlled in vitro conditions, while at the same time understanding contributing (patho-)mechanisms better.

Acknowledgement. We thank Michael Diedenhofen for excellent technical assistance with the image coregistration, and Jan Jikeli for help with the growth curve fits. We thank the Company Biospace for manufacturing the custom-built illumination platform. Generous supply of Endorem by Drs. C. Corot and P. Robert (Guerbet, France) is gratefully acknowledged. This study was supported by a grant from the Volkswagen-Stiftung, and by grants from the EU-FP6 program: DiMI (LSHB-CT-2005-512146) and EMIL (LSHC-CT-2004-503569).

Open Access. This article is distributed under the terms of the Creative Commons Attribution Noncommercial License which permits any noncommercial use, distribution, and reproduction in any medium, provided the original author(s) and source are credited.

\section{References}

1. Lin RZ, Chang HY (2008) Recent advances in three-dimensional multicellular spheroid culture for biomedical research. Biotechnol J 3 (9-10):1172-1184

2. Torisawa YS, Takagi A, Nashimoto Y, Yasukawa T, Shiku H, Matsue $\mathrm{T}$ (2007) A multicellular spheroid array to realize spheroid formation, culture, and viability assay on a chip. Biomaterials 28(3):559-566

3. Kaijzel EL, van der Pluijm G, Lowik CW (2007) Whole-body optical imaging in animal models to assess cancer development and progression. Clin Cancer Res 13(12):3490-3497

4. Henriquez NV, van Overveld PG, Que I, Buijs JT, Bachelier R, Kaijzel EL, Lowik CW, Clezardin P, van der Pluijm G (2007) Advances in optical imaging and novel model systems for cancer metastasis research. Clin Exp Metastasis 24(8):699-705

5. Bulte JW, Kraitchman DL (2004) Iron oxide MR contrast agents for molecular and cellular imaging. NMR Biomed 17(7):484-499

6. Gimi B, Mori N, Ackerstaff E, Frost EE, Bulte JW, Bhujwalla ZM (2006) Noninvasive MRI of endothelial cell response to human breast cancer cells. Neoplasia 8(3):207-213

7. Abe T, Wakimoto H, Bookstein R, Maneval DC, Chiocca EA, Basilion JP (2002) Intra-arterial delivery of p53-containing adenoviral vector into experimental brain tumors. Cancer Gene Ther 9(3):228-235

8. Nishikawa R, Ji XD, Harmon RC, Lazar CS, Gill GN, Cavenee WK, Huang HJ (1994) A mutant epidermal growth factor receptor common in human glioma confers enhanced tumorigenicity. Proc Natl Acad Sci USA 91(16): 7727-7731

9. Kustermann E, Himmelreich U, Kandal K, Geelen T, Ketkar A, Wiedermann D, Strecker C, Esser J, Arnhold S, Hoehn M (2008) Efficient stem cell labeling for MRI studies. Contrast Media Mol Imaging 3(1):27-37

10. Mansfield JR, Gossage KW, Hoyt CC, Levenson RM (2005) Autofluorescence removal, multiplexing, and automated analysis methods for in-vivo fluorescence imaging. J Biomed Opt 10(4):41207

11. Shaner NC, Campbell RE, Steinbach PA, Giepmans BN, Palmer AE, Tsien RY (2004) Improved monomeric red, orange and yellow fluorescent proteins derived from Discosoma sp. red fluorescent protein. Nat Biotechnol 22(12):1567-1572

12. Weissleder R, Ntziachristos V (2003) Shedding light onto live molecular targets. Nature Medicine 9:123-128 
13. Thorek DL, Tsourkas A (2008) Size, charge and concentration dependent uptake of iron oxide particles by non-phagocytic cells. Biomaterials 29(26):3583-3590

14. Shapiro EM, Skrtic S, Koretsky AP (2005) Sizing it up: cellular MRI using micron-sized iron oxide particles. Magn Reson Med 53(2):329-338

15. Shapiro EM, Medford-Davis LN, Fahmy TM, Dunbar CE, Koretsky AP (2007) Antibody-mediated cell labeling of peripheral $\mathrm{T}$ cells with micron-sized iron oxide particles (MPIOs) allows single cell detection by MRI. Contrast Media Mol Imaging 2(3):147-153

16. Brandl M, Tonn JC, Kotitschke K, Goldbrunner R, Kerkau S, Haase A (1995) Quantitative NMR microscopy of multicellular tumor spheroids and confrontation cultures. Magn Reson Med 34(4):596-603

17. Huang S, Vader D, Wang Z, Stemmer-Rachamimov A, Weitz DA, Dai G, Rosen BR, Deisboeck TS (2008) Using magnetic resonance microscopy to study the growth dynamics of a glioma spheroid in collagen I: a case study. BMC Med Imaging 8:3

18. Bernas LM, Foster PJ, Rutt BK (2007) Magnetic resonance imaging of in vitro glioma cell invasion. J Neurosurg 106(2):306-313

19. Hart LS, El-Deiry WS (2008) Invincible, but not invisible: imaging approaches toward in vivo detection of cancer stem cells. J Clin Oncol 26(17):2901-2910

20. Contag $\mathrm{CH}$ (2007) In vivo pathology: seeing with molecular specificity and cellular resolution in the living body. Annu Rev Pathol 2:277-305

21. Fischer U, Meese E (2007) Glioblastoma multiforme: the role of DSB repair between genotype and phenotype. Oncogene 26(56):7809-7815
22. Bao S, Wu Q, McLendon RE, Hao Y, Shi Q, Hjelmeland AB, Dewhirst MW, Bigner DD, Rich JN (2006) Glioma stem cells promote radioresistance by preferential activation of the DNA damage response. Nature 444(7120):756-760

23. Wild-Bode C, Weller M, Rimner A, Dichgans J, Wick W (2001) Sublethal irradiation promotes migration and invasiveness of glioma cells: implications for radiotherapy of human glioblastoma. Cancer Res 61(6):2744-2750

24. Thorsen F, Enger PO, Wang J, Bjerkvig R, Pedersen PH (2007) Human glioblastoma biopsy spheroids xenografted into the nude rat brain show growth inhibition after stereotactic radiosurgery. J Neurooncol 82(1): $1-10$

25. McCaffrey A, Kay MA, Contag CH (2003) Advancing molecular therapies through in vivo bioluminescent imaging. Mol Imaging 2 (2):75-86

26. Gross S, Abraham U, Prior JL, Herzog ED, Piwnica-Worms D (2007) Continuous delivery of D-luciferin by implanted micro-osmotic pumps enables true real-time bioluminescence imaging of luciferase activity in vivo. Mol Imaging 6(2):121-130

27. Qiao Y, Spitz MR, Guo Z, Hadeyati M, Grossman L, Kraemer KH, Wei Q (2002) Rapid assessment of repair of ultraviolet DNA damage with a modified host-cell reactivation assay using a luciferase reporter gene and correlation with polymorphisms of DNA repair genes in normal human lymphocytes. Mutat Res 509 (1-2):165-174 